\title{
Comunidades de aranhas (Araneae) em cultivos de seringueira (Hevea brasiliensis Muell. Arg.) no Estado de São Paulo
}

\author{
Isabela Maria Piovesan Rinaldi ${ }^{1}$ \\ Gustavo Rodrigo Sanches Ruiz ${ }^{2}$
}

\begin{abstract}
Spider communities (Araneae) on rubber tree (Hevea brasiliensis Muell. Arg.) plantations in São Paulo State, Brazil. The spider fauna composition of three rubber tree commercial plantations in the Northwest part of São Paulo State, Brazil, was characterized for both canopy and litter strata. On seven occasions from April 2000 to October 2001, samples were taken by beating sheet and hand capture, resulting in a total of 946 individuals, belonging to 24 families and 119 species. The most common species were Italaman santamaria Brescovit, 1997 and Teudis sp. (Anyphaenidae), Castianeira sp. and Falconina aff. gracilis (Corinnidae), Paracleocnemis sp. (Philodromidae), Ibotyporanga naideae Mello-Leitão, 1944 (Pholcidae), Chira spinipes (Taczanowiski, 1871) and Rudra sp. (Salticidae), Achaearanea hirta (Taczanowiski, 1873) and Coleosoma floridanum (Banks, 1900) (Theridiidae) and Goeldia sp. (Titanoecidae). Anyphaenidae, Theridiidae and Salticidae were the most abundant families in the canopy, while Pholcidae and Corinnidae in the litter. Spider abundance was found to be, in general, positively correlated to the litter volume and density of branches in the trees. The use of acaricides and insecticides for one plantation resulted in a decrease in spider abundance for both strata. Abundance values among the samples suggest that the canopy spider abundance decreases with the loss of leaves in the dry season. Simultaneously, litter spider abundance increased in this season, because of the increase in litter volume. The most active canopy spiders, like runners and stalkers, should be investigated for their potential as pest control agents. KEY WORDS. Spiders, Hevea brasiliensis, guilds, biodiversity, pesticides
\end{abstract}

Aranhas compõem um dos grupos de predadores nativos mais abundantes na maioria dos habitats terrestres, incluindo os agrícolas. Como inimigos naturais de artrópodes, as aranhas podem contribuir na regulação das populações de insetos e de ácaros fitófagos, prejudiciais para cultivos comerciais. Por esta razão, as aranhas têm sido ativamente preservadas em áreas agrícolas da China (ZHAO 1993 apud MARC \& CANARD 1997).

Estudos sobre biodiversidade e ecologia básica desses predadores em agroecossistemas são fundamentais para uma utilização mais racional do ambiente pelo homem. Levantamentos conduzidos em áreas agrícolas têm revelado alta diversidade de aranhas (RINALDI \& FORTI 1997), o que afasta a idéia freqüente de que os sistemas agrícolas são simplificados quanto à araneofauna.

1) Departamento de Zoologia, Instituto de Biociências, Universidade Estadual Paulista. Caixa Postal 510, 18618-000 Botucatu, São Paulo, Brasil. E-mail: rinaldi@ibb.unesp.br

2) Departamento de Zoologia e Botânica, Institulo de Biociências, Letras e Ciências Exatas, Universidade Estadual Paulista. Caixa Postal 136, 15054-000 São José do Rio Preto, São Paulo, Brasil. Bolsista FAPESP. E-mail: gustavoruiz86@hotmail.com 
Hevea brasiliensis Muell. Arg., Euphorbiaceae, tem grande importância econômica na produção de látex. Seu cultivo na região noroeste do Estado de São Paulo encontra-se em franca expansão segundo dados da "Associação Paulista de Produtores e Beneficiadores de Borracha".

Este estudo tem o objetivo de caracterizar as comunidades de aranhas presentes em seringais, até agora desconhecidas, determinando a abundância, a composição de famílias, a diversidade de espécies e grupos ecológicos (guildas), tanto na copa das árvores quanto no folhedo, bem como, comparar as comunidades dos diferentes cultivos.

\section{MATERIAL E MÉTODOS}

Os cultivos de $H$. brasiliensis estudados localizam-se nos municípios de

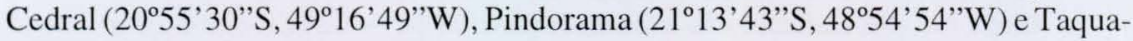
ritinga ( $21^{\circ} 26^{\prime} 39^{\prime \prime}$ 'S $\left.48^{\circ} 37^{\prime} 52^{\prime \prime} \mathrm{W}\right)$, no noroeste do Estado de São Paulo. No cultivo de Taquaritinga administram-se constantemente os acaricidas-inseticidas deltamethrin, monocrotophos e propargite.

O clima da região noroeste do Estado de São Paulo caracteriza-se por apresentar duas estações, uma úmida (de outubro a março), com temperatura média de $26,4^{\circ} \mathrm{C}$, e outra seca (de abril a setembro), com temperatura média de $21,0^{\circ} \mathrm{C}$ (BARCHA \& ARID 1971). O volume de precipitação da estação úmida representa $85 \%$ da precipitação total anual.

Foram realizadas sete coletas trimestrais, entre abril de 2000 e outubro de 2001. A cada coleta foram amostradas, aleatoriamente, três árvores em cada área de cultivo, totalizando, ao final do experimento, 63 árvores.

O método de coleta utilizado para amostrar a copa das árvores foi a batida, mediante um cabo telescópico, de todos os galhos inferiores a $4 \mathrm{~m}$ de altura, sendo os mais baixos sacudidos manualmente, com violência, sobre um lençol de $5 \mathrm{~m}^{2}$. Para amostragem do folhedo, foram capturados por dois coletores os artrópodes presentes nas folhas, frutos e solo fragmentado, e também aqueles habitantes da base do tronco, numa área de $1 \mathrm{~m}^{2}$, cercada por uma moldura de madeira, ao redor de cada árvore alvo, com esforço de coleta padronizado em 15 minutos.

O material está depositado na coleção científica do Departamento de Zoologia do Instituto de Biociências da UNESP, Campus de Botucatu, São Paulo. A caracterização das guildas, ou seja, da exploração similar de recursos similares por grupos de espécies, foi baseada em UETZ et al. (1999) e em observações de campo. A superguilda das aranhas caçadoras foi dividida em caçadoras cuspideiras (Scytodidae), caçadoras por emboscada (Philodromidae, Selenopidae e Thomisidae), caçadoras perseguidoras (Salticidae), caçadoras de solo (Caponiidae, Corinnidae, Dipluridae, Gnaphosidae, Lycosidae, Miturgidae, Segestriidae e Zodariidae) e caçadoras de folhagens (Anyphaenidae, Senoculidae e Sparassidae), enquanto a superguilda das aranhas tecelãs foi dividida em tecelãs orbiculares (Araneidae e Tetragnathidae), tecelãs errantes (Linyphiidae), tecelãs tridimensionais (Dictynidae, Pholcidae e Theridiidae) e tecelãs em lençol (Filistatidae e Titanoecidae). A inclusão de Dipluridae na superguilda das caçadoras é justificada pelo fato de que a única espécie coletada não apresentou hábitos de Dipluridae tecelãs. 
A diversidade e a uniformidade da araneofauna de cada cultivo foram calculadas pelos índices de Shannon-Wiener ( $\left.\mathrm{H}^{\prime}, \log _{10}\right)$ e de Pielou (e), respectivamente (ODUM 1988).

\section{RESULTADOS}

Foram coletados 946 indivíduos, pertencentes a 24 famílias e 119 espécies (Tab. I), com base em espécimes adultos e imaturos.

Onze espécies representaram 52\% $(\mathrm{N}=493)$ das aranhas coletadas, a saber, Italaman santamaria Brescovit, 1997 e Teudis sp. (Anyphaenidae), Castianeira sp. e Falconina aff. gracilis (Keyserling, 1891) (Corinnidae), Paracleocnemis sp. (Philodromidae), Ibotyporanga naideae Mello-Leitão, 1944 (Pholcidae), Chira spinipes (Taczanowiski, 1871) e Rudra sp. (Salticidae), Achaearanea hirta (Taczanowiski, 1873), Coleosoma floridanum (Banks, 1900) (Theridiidae) e Goeldia sp. (Titanoecidae).

A biodiversidade de famílias e espécies de aranhas no folhedo foi sempre maior que na copa, nos três cultivos. O número de famílias de aranhas foi maior nos cultivos de Cedral e de Taquaritinga do que no de Pindorama, e o maior número de espécies foi observado no cultivo de Cedral, seguindo-se os de Taquaritinga e Pindorama. Já a abundância total foi maior no cultivo de Pindorama, seguido pelos de Cedral e Taquaritinga. A abundância variou entre os dois estratos dos cultivos, ora sendo maior na copa do que no folhedo (Cedral), ora o inverso (Pindorama), ou ainda iguais (Taquaritinga). A diversidade e a equitabilidade de espécies foram inversamente proporcionais à abundância (Tab. I).

Tabela I. Abundância, número de famílias e de espécies de aranhas e índices de diversidade e de equitabilidade nos três cultivos de Hevea brasiliensis na região noroeste do Estado de São Paulo.

\begin{tabular}{|c|c|c|c|c|c|c|}
\hline Cultivo & Estrato & Número familias & Número espécies & Abundância & $\mathrm{H}^{\prime}$ & e \\
\hline \multirow[t]{3}{*}{ Cedral } & Copa & 11 & 33 & 232 & 1,025 & 0,675 \\
\hline & Folhedo & 15 & 39 & 140 & 1,319 & 0,829 \\
\hline & Total & 19 & 66 & 372 & 1,427 & 0,784 \\
\hline \multirow[t]{3}{*}{ Pindorama } & Copa & 10 & 23 & 179 & 0,995 & 0,731 \\
\hline & Folhedo & 17 & 40 & 210 & 1,035 & 0,646 \\
\hline & Total & 17 & 52 & 389 & 1,238 & 0,718 \\
\hline \multirow[t]{3}{*}{ Taquaritinga } & Copa & 6 & 17 & 93 & 0,970 & 0,788 \\
\hline & Folhedo & 17 & 44 & 92 & 1,507 & 0,911 \\
\hline & Total & 19 & 56 & 185 & 1,545 & 0,884 \\
\hline Total & & 24 & 119 & 946 & & \\
\hline
\end{tabular}

Das 24 famílias, 14 foram responsáveis por $95 \%$ a $99 \%$ do total de indivíduos em cada localidade, e ocorreram nos três cultivos (Fig. 1). Na comparação entre os cultivos, apenas duas ou três famílias foram exclusivas de cada um (Fig. 1).

Anyphaenidae, Salticidae e Theridiidae foram as famílias mais abundantes em todos os cultivos, acrescentando-se Pholcidae para Pindorama. No cultivo de Cedral, as famílias Anyphaenidae, Salticidae, Theridiidae e Corinnidae, em ordem decrescente de freqüência, somaram $75 \%$ da araneofauna. As mesmas famílias 


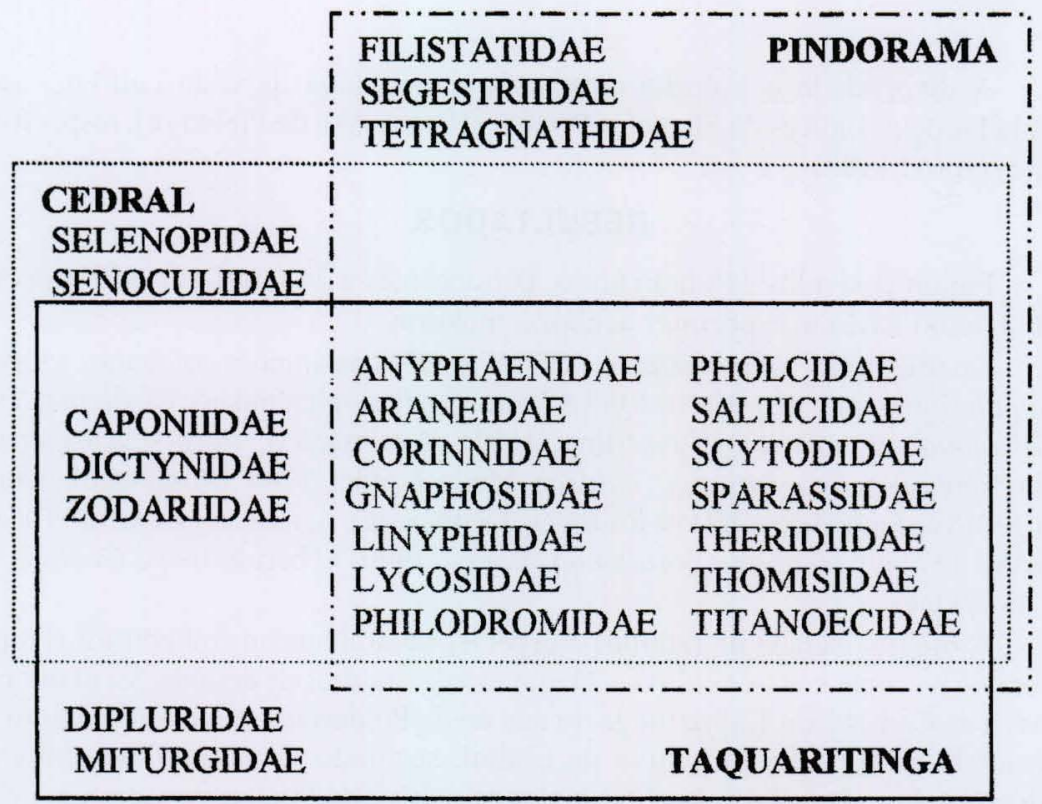

Fig. 1. Co-ocorrência de famílias de aranhas nos três cultivos de Hevea brasiliensis na região noroeste do Estado de São Paulo.

citadas, acrescidas de Pholcidae, entre as duas primeiras, representaram $81 \%$ das aranhas no cultivo de Pindorama. No cultivo de Taquaritinga, as famílias mais freqüentes foram Theridiidae, Salticidae e Anyphaenidae, somando 67\% do total de aranhas encontradas (Fig. 2).

A araneofauna da copa teve os maiores picos de abundância no início das estações secas (abril), diferentemente da fauna do folhedo, que teve picos nas estações úmidas dos dois anos de amostragem nos cultivos de Cedral e Pindorama. No cultivo de Taquaritinga, a abundância foi menor que nos demais cultivos, tanto na copa quanto no folhedo, apresentando, este último, abundância baixa e constante (Fig. 3).

Foram evidenciadas nove categorias de guildas de aranhas, cinco de caçadoras e quatro de tecelãs (Fig. 4). Em todos os cultivos, predominaram as aranhas caçadoras de folhagem (principalmente Anyphaenidae), as tecelãs tridimensionais (Theridiidae e Pholcidae) e as perseguidoras (Salticidae). Anyphaenidae foi a principal aranha da copa nos três cultivos.

Toda a árvore é colonizada por aranhas, incluindo os artefatos usados na exploração do látex. A. hirta e I. naideae ocuparam com grande freqüência os espaços entre os copos coletores e os troncos das árvores. A copa abrigou principalmente aranhas caçadoras de folhagem, isto é, aranhas corredoras de curtas distâncias, que capturam presas com grande agilidade e rapidez à noite. Já no folhedo, predominaram tecelãs tridimensionais e caçadoras de solo (principalmente Corinnidae). Registrou-se intensa colonização das cascas secas dos frutos de $H$. brasiliensis caídos no solo por I. naideae e, com menor freqüência, por Goeldia sp. (Fig. 5). 


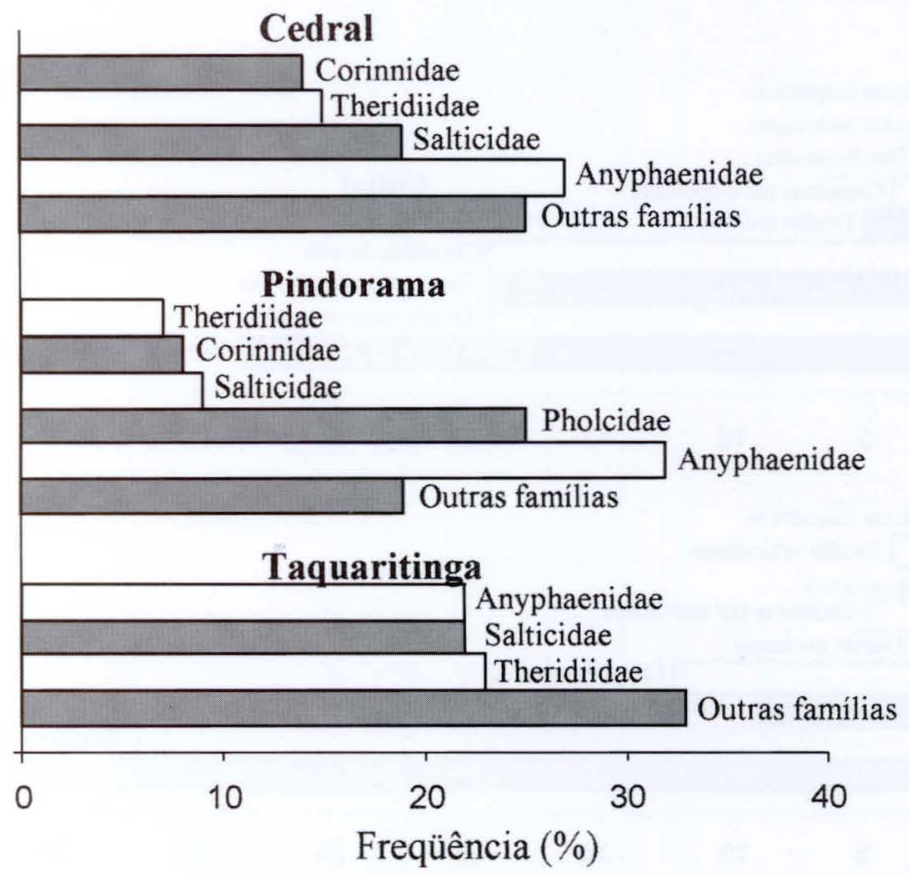

Fig. 2. Distribuição das familias mais freqüentes nos três cultivo de Hevea brasiliensis na região noroeste do Estado de São Paulo.

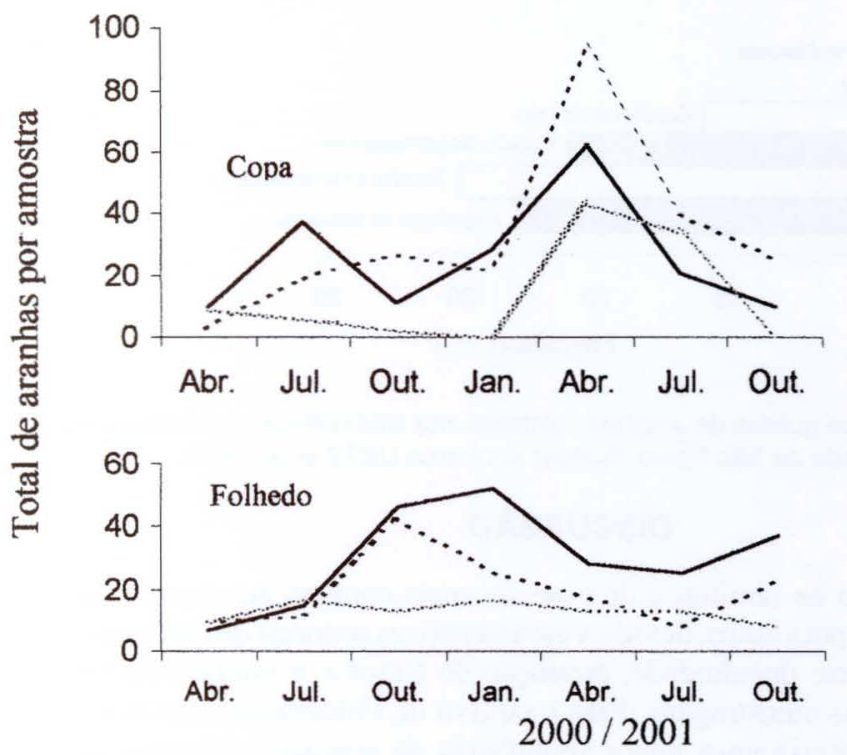

Fig. 3. Abundância total de aranhas nos três cultivos de Hevea brasiliensis na região noroeste do Estado de São Paulo ao longo das amostras. 


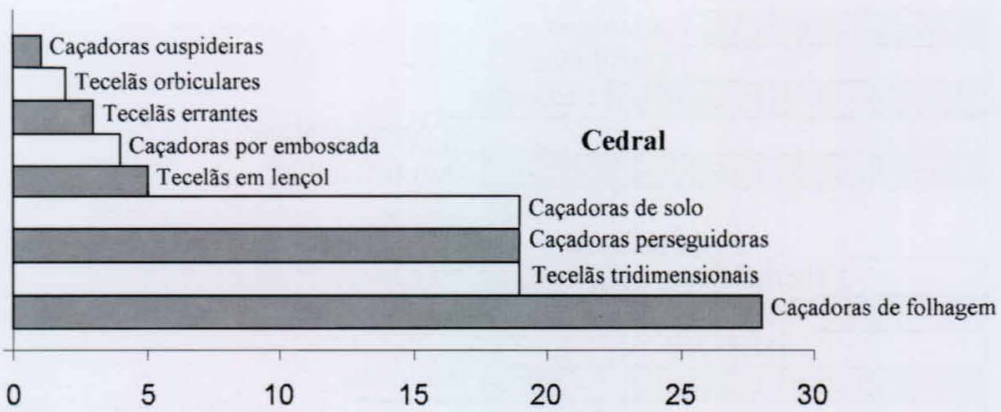

Caçadoras cuspideiras Tecelãs orbiculares

Tecelãs errantes Pindorama

Caçadoras por emboscada

Tecelãs em lençol

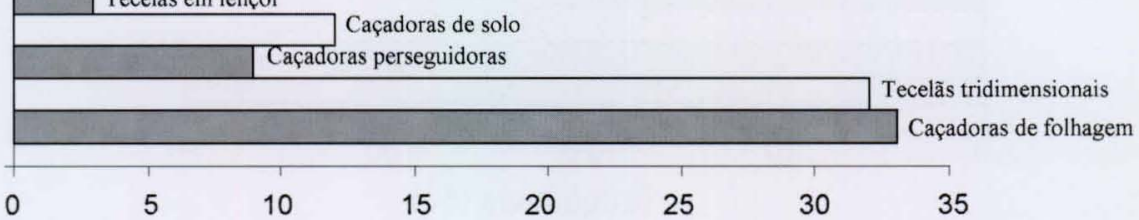

Caçadoras cuspideiras Tecelãs orbiculares

Taquaritinga

Tecelãs errantes

Caçadoras por emboscada

Tecelãs em lençol

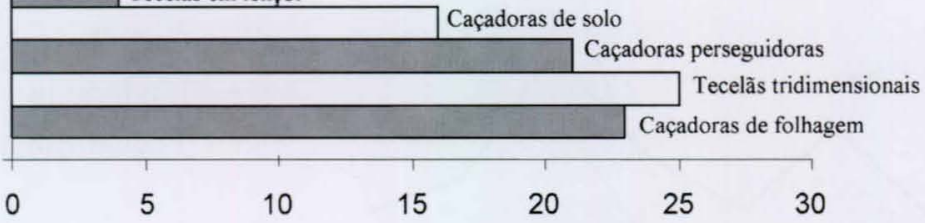

Freqüência (\%)

Fig. 4. Freqüência (\%) de guildas de aranhas coletadas nos três cultivos de $H$. brasiliensis na região noroeste do Estado de São Paulo. Guildas conforme UETZ et al. 1999.

\section{DISCUSSÃO}

A composição de famílias e de espécies mais comuns nas áreas estudadas variou de um cultivo para outro, devido a características próprias de cada um, como a quantidade de ramos, deciduidade, produção de frutos e o volume do folhedo, observados durante as amostragens. Para o cultivo de Pindorama, com árvores de copas mais densas, registramos maior abundância de aranhas habitantes de copa (Fig. 4), certamente pela maior quantidade de microhabitats que representam, conforme observado por vários autores, dentre eles HATLEY \& MACMAHON (1980). 


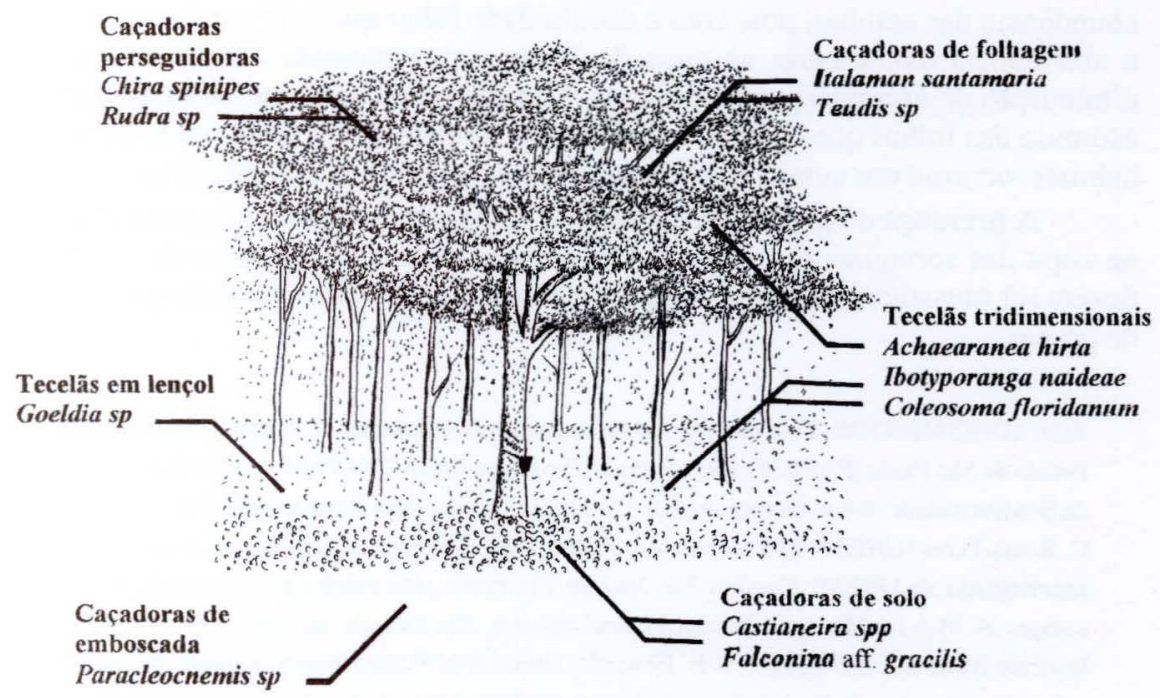

Fig. 5. Ocorrência das espécies mais freqüentes de aranhas e suas guildas em cultivos de seringueira.

Também no cultivo de Pindorama, observou-se I. naideae como a espécie mais abundante, ocupando o grande número de frutos secos presentes no folhedo. O volume e estrutura do folhedo foram reportados por vários autores (UETZ 1979; BULTMAN et al. 1982; JENNINGS et al. 1988) como fatores que influenciam positivamente a abundância de aranhas.

O uso de defensivos no cultivo de Taquaritinga foi provavelmente o principal responsável pela menor abundância de aranhas. RIECHERT \& LOCKLEY (1984) afirmaram que os defensivos constituem a maior causa de mortalidade desses artrópodes. Contudo, o cultivo de Taquaritinga apresentou os maiores valores de diversidade e de equitabilidade (Tab. I). Esses valores, calculados separadamente para copa e folhedo, possibilitaram a conclusão de que a alta diversidade no cultivo tratado deveu-se à fauna do folhedo, pois os inseticidasacaricidas diminuem a abundância e, consequentemente, a riqueza na copa (Tab. I), e provavelmente também no folhedo. A fauna edáfica de áreas vizinhas ao cultivo tratado tende a se deslocar para o folhedo do seringal, aumentando a riqueza, mas ao contrário de se estabelecer, tende a morrer por ação dos defensivos, resultando em espécies com poucos indivíduos. A queda na abundância das espécies tem como conseqüência uma queda na dominância, aumentando a diversidade no folhedo $\left(\mathrm{H}^{\prime}=1,507, \mathrm{e}=0,911\right)$. 
Em todos os cultivos, a diversidade da fauna no folhedo foi sempre maior que a diversidade na copa. A fenologia das seringueiras afetou o padrão de abundância das aranhas, pois com a deciduidade foliar que ocorre na estação seca, a abundância das aranhas na copa declinou, provavelmente em decorrência da diminuição de esconderijos e de presas. Simultaneamente como consequiência do acúmulo das folhas que caem na estação seca, aumentando a quantidade de microhabitats, ocorreu um aumento na abundância de aranhas no folhedo (Fig. 3).

A presença de aranhas caçadoras de folhagem, e também as perseguidoras na copa das seringueiras, por sua alta freqüência e estratégia ativa de predação, devem ser investigadas como potenciais agentes no controle natural das populações de pragas.

AGRADECIMENTOS. Este trabalho foi financiado pela Fundação de Amparo à Pesquisa do Estado de São Paulo (FAPESP) como parte do Programa BIOTA/FAPESP - O Instituto Virtual da Biodiversidade (www.biotasp.org.br). Os autores agradecem a Reinaldo J.F. Feres e Denise C. Rossa-Feres (UNESP- São José do Rio Preto) pelas sugestões ao manuscrito, e à equipe de acarologistas da UNESP, Campus São José do Rio Preto, pela colaboração nos trabalhos de campo. A M.A.L. Marques, Fundação Zoobotânica, Rio Grande do Sul; A.D. Brescovit, Instituto Butantan, São Paulo e G.B. Edwards, Division of Plant Industry, Gainesville, EUA, pelas identificações de Theridiidae e Pholcidae, Clubionoidea e Salticidae, respectivamente. A J.M. Pisani pelo desenho.

\section{REFERÊNCIAS}

BARCHA, S.F. \& F.M. ARID. 1971. Estudo da evapotranspiração na região Norte-Ocidental do Estado de São Paulo. Revta Cient. Faculd. Ciênc. Letras Votuporanga 1: 97-122.

BULTMAN, T.L.; G.W. UETZ; A.R. BRADY. 1982. A comparison of cursorial spider communities along a successional gradient. Jour. Arachnol., New York, 10: 23-33.

HATLEY, C.L. \& J.A. MACMAHON. 1980. Spider community organization: seasonal variation and the role of vegetation architecture. Environ. Entomol., Richmond, 9: 632-39.

JENnings, D.T. \& D.J. HiLbuRn. 1998. Spiders (Araneae) captured in Malaise traps in spruce-fir forests of west-central Maine. Jour. Arachnol., New York, 16: 85-94.

MARC, P. \& A.CANARD. 1997. Maintain spider biodiversity in agroecosystems as a tool in pest control. Agriculture, Ecosystems and Environment, Amsterdam, 62: 229 - 235.

Odum, E. 1988, Ecologia. Rio de Janeiro, Editora Guanabara, XI+434p.

RIECHERT, S.E. \& T. LOCKLEY. 1984. Spiders as biological control agents. Ann. Rev. Entomol., Stanford, 29: 299-320.

RINALDI, I.M.P. \& L.C. FoRTI. 1997. Hunting Spiders of Woodland Fragments and Agricultural Habitats in the Atlantic Rain Forest Region of Brazil. Stud Neotrop Fauna \& Environm, Tübingen, 32: 244-255.

Uetz, G.W. 1979. The influence of variation in litter habitats on spider communities. Oecologia, Berlin, 40: $29-42$.

Uetz, G.W.; J. HalaJ \& A B. Cady. 1999. Guild structure of spiders in major crops. Jour. Arachnol., New York, 27: 270-280.

Recebido em 08.IV.2002; aceito em 15.VIII.2002. 